\title{
Antnet Algorithm in Route Searching on Computer Networks
}

\author{
Harvei Desmon Hutahaean ${ }^{1}$, R. Mahdalena Simanjorang ${ }^{2}$, Penda Sudarto Hasugian ${ }^{3}$ \\ \{harvei11@unimed.ac.id ${ }^{1}$, relimamahdalenasimanjorang@yahoo.co.id ${ }^{2}$, penda.hasugian@gmail.com $\left.{ }^{3}\right\}$ \\ ${ }^{1}$ Department of Electrical Engineering, Universitas Negeri Medan, Medan, Indonesia \\ ${ }^{2,3}$ STMIK Pelita Nusantara Medan, Indonesia
}

\begin{abstract}
The speed of technological development makes the process of transformation of information as a major human need that will be easily obtained with an increasingly broad range of computer network developments following the revolution that occurs in Personal Computers. With the existence of computer technology as a support in data processing, information has become a necessity, the information needed must be obtained quickly and accurately. In fact, one of the most important factors in information technology is the network or media access which is the bridge between sources of information for various parties. Network characteristics such as traffic load and topology on a network can change dynamically. The Antnet algorithm is a multi-agent distribution system that is inspired by the stimulation communication model carried out in ant colonies. The algorithm is implemented in the search for the shortest route on a computer network, the search for the shortest route on a computer network is used to get the fastest and without delay.
\end{abstract}

Keywords: Shortest Route, Computer Network, Antnet Algorithm.

\section{Introduction}

The speed of technological development makes the process of information transformation as a major human need that will be easily obtained with a broader scope of development of computer networks following the revolution that occurs on PCs. Mass production of Personal Computer makes ownership of this device easier and cheaper. Local Area Network (LAN) is evolving to accompany the PC development revolution. Increased LAN capability in exchanging files and messages between computers in a relatively small geographic area. Other computers can share resources and act as file providers, bus topologies connecting each computer / node with a communication cable through a a computer interface card. Every computer can be connected to other computers on the network, meaning all computers have the same position on the network and are not dependent on a central server computer. When you want to access other computers in a computer network, you only need to know the address of the computer / node to be addressed to and can directly communicate and also different ring, star, mesh, and tree topologies. The ring topology is connected to a single cable and forms part of a ring and the data is sent across several existing nodes to the destination node. Whereas the star topology of several computers will be connected to one computer center and different from the mesh topology has a double path at each node / network node and tree topology is a combination of star topology and bus topology and because of that LAN network and BUS topology are very suitable in the search for the shortest route. 
Finding the shortest route on a computer network is very important. The reason for searching the shortest route on a computer network is to get the results of the quickest route and without delay, because it requires a way to overcome the problem. This shortest route search is often called the router algorithm. There are so many algorithms that can be used to solve this problem like the AntNet algorithm. In solving the shortest route problem, this algorithm has several advantages which are discussed further in this research. This study aims to analyze the Antnet algorithm in solving problems determining the search for the shortest route on a computer network. So with this script, it is hoped that it can be concluded that the right antnet algorithm for searching the shortest route on a computer network.

Where has a large influence on the overall performance of a network. Network characteristics such as traffic load and topology on a network can change dynamically. This research proposes the use of the AntNet algorithm to overcome the optimization of data packet delivery. Antnet is a multi-agent distribution system that is inspired by the stimulation communication model carried out in ant colonies. From the simulation results, AntNet shows a better performance than the Dijkstra algorithm on the performance of the results of the packet throughput and time delay. There are several types of routing problems, one of which is the nature of distribution, so a solution that assumes access to all forms of global information is not wanted.

\section{Routing}

Routing is the process of moving data across a network by going through several network segments using equipment called a router. Router (route regulator) will choose the right data path according to the direction of the data destination. Placement of routers on the network will combine and connect small routers that will form an entity called between networks or interworks.

The router will manage information about the direction of the data path from a file into a scheme called a route table. This table contains network router (or port) interface information that is used to send data through certain network segments. The router will not run broadcast packets with no known purpose. The router will manage a packet that is sent if it has a specific purpose.

\section{Antnet Algorithm}

The AntNet algorithm uses two mobile agents called forward ants and backward ants to update the value of the routing table. Forward ants use the heuristic method in the routing table to move towards the destination past existing nodes and get information about the distribution of traffic on a network. Backward ants use the path from the forward ants used in the opposite direction. At each node, the backward ants update the routing table and additional tables that contain the distribution of traffic on the network. AntNet has been known to have better results than OSPF, Bellman-Ford, etc. routing protocols with various and close traffic loads. For example a network with graph $\mathrm{G}(\mathrm{N}, \mathrm{L})$ contains $\mathrm{N}$ nodes and L paths. All lines on the network are bidirectional where the transmission capacity and transmission delay have been determined. Each node is assumed to be a communication between end-point (host) and router.

Each node of the network maintains an input buffer composed of a single queue and output buffer is composed of high priority queues and low priority queues for each neighbor node or output path. High priority queues are served before low priority queues. All packets on the network can be divided into two classes: 
a) Data packet: shows information about exchanges between hosts with each other. In ant-routing, data packets do not maintain routing information, ant-routing use information that has been stored in the routing table to roam from the origin source to the destination node.

b) Mobile agents (forward ants and backward ants): used to update routing tables and distribute information about traffic loads on the network.

\section{Research Method}

AntNet is divided into two homogeneous mobile agents called forward ants and backward ants. These agents have the same nature, it's just different placement of the situation and environment, so they can receive different inputs and produce different outputs. In general, these ant agents can be classified as deliberative, because they have the behaviour to regenerate again and at the same time they can maintain a complex internal status. Each agent communicates indirectly or staggery. Agents capture the information they read and write the information obtained in the two data structures stored at each network node as shown in Figure 1 below

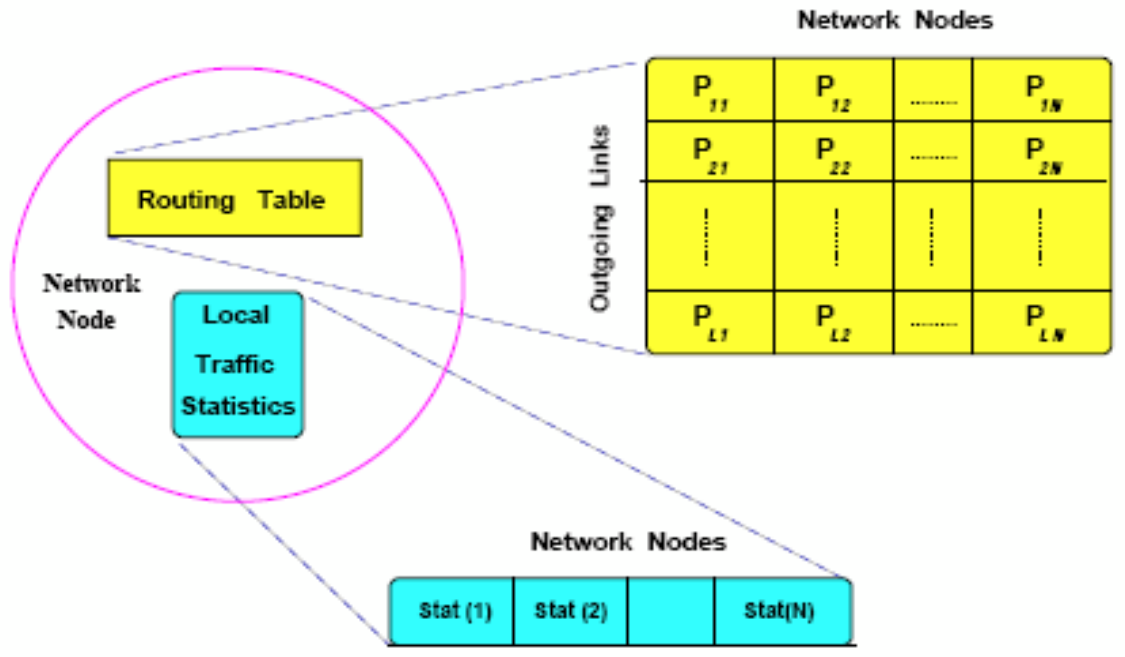

Fig 1. Data Structure Stored By AntNet 
A layer can communicate "vertically" with another layer that is directly below or above it.

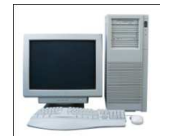

Computer 1

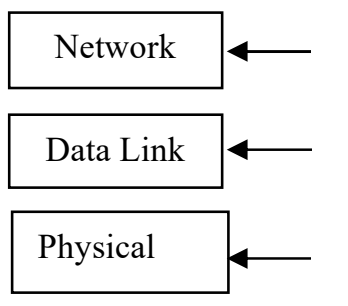

Vertical Communication

Vertical Communication

Vertical Communication

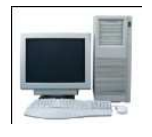

Computer 2

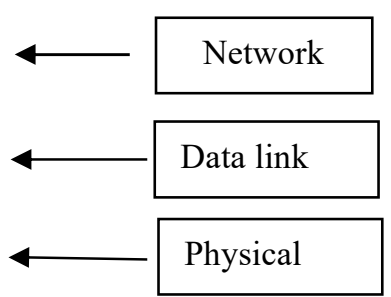

Fig 2. Inter-Layer Communication Process

Information flowing from one layer to another will experience transformation. To understand this, consider the following illustration that illustrates the transformation of information from the Application layer to the Physical layer.

1. Information starts from the Application layer and then it passes through the Presentation layer and the Session layer. At this stage data transformation is not done yet. Information through these three layers is called PDU (Protocol Data Unit) or data only.

2. After arriving at the Transport layer, the data will be transformed into another form called a segment.

3. Segments flow to the network layer and then convert into packets (sometimes called datagrams).

4. Packets flow to the Data Link layer and then convert into frames.

5. Frame flows to the Physical layer and then converted into bits converted into physical quantities, such as electric current, electromagnetic waves.

The process of "changing shape" from one layer to the next is done by adding a special header, this is what is called encapsulation. The encapsulation process becomes repetitive until the data is converted into bits. Then these bits are sent to the target host via network media. After the information (changed bits) reaches the target host, the reverse process, which is to release the header one by one from the layer carried up to the top layer will be done, this process of removing the header is called de-encapsulation. The process of encapsulation and deencapsulation can be analogized to sending goods via post. Items sent will be wrapped, given an address, delivered to the destination address. After arriving at the destination, the recipient can open the package again.

\section{Result and Discussion}

The ant algorithm is adopted from the behavior of the ant colony known as the ant system (Dorigo, 1996). An ant colony is naturally able to find the shortest route on the way from the nest to the places where food sources, based on footprints on the track that has been passed. The 
more ants that pass through a track, the clearer the footprints. This causes the path the ants traverse in small amounts the less the ant density that passes through it, or it will not even be crossed at all. We recommend that the trajectory that is passed by ants in large quantities the longer will increase the density of ants that pass through it or even all ants pass through that path. There are two groups of ants that travel, L group of ants depart from left to right and group of ants $\mathrm{R}$ depart from right to left. Both groups depart from the same point and in the decision making position which way to take, group L divides two groups again. Some pass through the upper road and some pass through the lower road, this also applies to group $\mathrm{R}$. The pheromones left behind by the ants that pass through the upper road are experiencing a lot of evaporation because the ants that pass the upper path are few compared to the path below. This is due to the distance traveled longer than the road below, whereas the pheromones that are at the bottom of the evaporation tend to be longer. The other ants finally decide to go down the road because there are already a lot of pheromones left, while the pheromones on the upper road have evaporated so much that the ants don't choose the upper way. The more ants that pass through the road, the more ants that follow it, the fewer ants that pass through the road, the pheromones left behind are reduced and even disappear. From this, the shortest path between nests and food sources was chosen.

\section{Step 1}

Initialize the price of algorithm parameters, initialized parameters are:

1. The intensity of the ant trail between the city and its changes $\left(t_{i j}\right)$

2. Many cities ( $\mathrm{n}$ ) including $\mathrm{x}$ and $\mathrm{y}$ (coordinates) or $d_{i j}$ (distance between networks)

3. Determination of networks and goals

4. Tetapan siklus semut (Q)

5. The control set of ant trail intensity $(\alpha)$

6. Visibility control settings $(\beta)$

7. Visibility between cities $=\left(1 / d_{i j}\left(\eta_{i j}\right)\right.$

8. Number of ants (m)

9. Evaporation constant trace ants $(\rho)$

10. Maximum number of cycles $\left(N C_{\max }\right)$ is fixed as long as the algorithm is run, meanwhile $t_{i j}$ will always be updated in value at each cycle of the algorithm starting from the first cycle $(\mathrm{NC}=1)$ until the maximum number of cycles is reached $\left(\mathrm{NC}=N C_{\max }\right)$ or until convergence occurs.

The Antnet testing parameter is:

Tij $=0,1$

Ants $=25$

$\propto=1, \quad \beta=1, \quad \mathrm{Q}=1, \quad \rho=0,5, \quad \mathrm{n}=3, \quad \operatorname{dij}=10, \quad \mathrm{xi}=4, \quad \mathrm{xj}=2, \quad \mathrm{yi}=6, \quad \mathrm{yj}=3$

\section{Step 2}

Fill out the first city into the tabu list. The results of the initialization of the first ant city in step 1 must be filled in as the first element of the tabu list. The result of this step is to fill in the first tabu list element of each ant with the first network index.

\section{Step 3}

Preparation of the path of each ant visit to each network. The ant colony that has been distributed to the first city will start traveling from the first city as the city of origin and one of the other cities as the destination city, and then from the second city, each ant colony will 
proceed by selecting one of the cities that is not on $t a b u_{k}$ as the next destination the ant colony journey continues until it reaches the city that has been determined. If s states the order of visit, the city of origin is stated as $\operatorname{tahu}_{k}(\mathrm{~s})$ and other cities are declared as $\left\{\mathrm{N}-\operatorname{tah} u_{k}\right\}$, then to determine the destination city used the probability equation of the city to be visited as follows, With $d_{i j}$ is the distance between city $\mathrm{i}$ and city $\mathrm{j}$ calculated based on the equation;

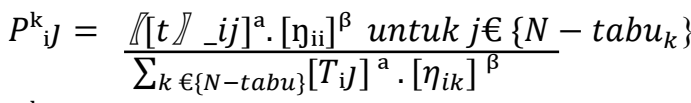

$P_{\mathrm{i} J}^{\mathrm{k}}=0$, for $\mathrm{j}$

where $\mathrm{i}$ is the origin word index and $\mathrm{j}$ is the destination city index.

$$
\begin{aligned}
P^{\mathrm{k}}{ }_{\mathrm{i} J} & =\frac{[0,1]^{1} \cdot[1 / 10]^{1}}{\sum^{\mathrm{k}^{\prime}} €\left\{N-t a b u[t i k]^{\alpha} \cdot\left[\mathrm{\eta}_{\mathrm{ii}}\right] \beta\right.} \\
0= & \frac{[0,1]^{1} \cdot[0,1]^{1}}{\sum_{-}\left({ }^{\mathrm{k}} €\{N-t a b u)^{\prime}[t i k]^{\alpha} \cdot\left[\mathrm{\eta}_{\mathrm{ii}}\right]\right.} \beta \sum[t i k]^{\alpha} .
\end{aligned}
$$

$\sum_{\mathrm{k}^{\prime} \in\{N-t a b u}[t i k]^{\alpha} \cdot[\text { nik }]^{\beta}=0,1 \cdot 0,1$

then

$\sum_{\mathrm{k}^{\prime}} €\left\{N-t a b u[t i k]^{\alpha} .[\text { nik }]^{\beta}=0,01\right.$

\section{Step 4}

a. Path length calculation of each ant

Calculation of the length of a closed path (length closed tour) or $L_{k}$ each ant is done after one cycle is completed by all ants. Calculations are based on $\operatorname{tabu}_{k}$.

b. Shortest route search

After $L_{k}$ is calculated, a minimum path length will be obtained for each cycle or $L_{\operatorname{minNC}}$ and the minimum price of the total length of the closed lane is or $L_{\text {min- }}$

c. Calculation of changes in the price of ant footprint intensity between cities.

The ant colony will leave footprints on the path between the city in its path. Evaporation and differences in the number of ants that pass, causing the possibility of changes in the price of the intensity of ant footprints between cities. The equation of change is

$$
\begin{aligned}
d_{i j} & =\sqrt{\left(x_{\mathrm{i}}-x_{j}\right)^{2}+\left(y_{i}-y_{j}\right)^{2}} \\
d_{i j} & =\sqrt{\left(x_{\mathrm{i}}-x_{j}\right)^{2}+\left(y_{i}-y_{j}\right)^{2}} \\
& =\sqrt{(4-2)^{2}+(6-3)^{2}} \\
& =\sqrt{2^{2}+3^{2}} \\
& =\sqrt{4+6} \\
d_{i j} & =\sqrt{10}
\end{aligned}
$$

then 


$$
\begin{aligned}
\Delta t_{i j} & =\Delta t_{y}=\sum_{k-1}^{m} \Delta t_{i j}^{k} \\
\Delta t_{i j} & =\Delta t_{y}=\sum_{k-1}^{25} \Delta t_{i j}^{k} \\
\Delta t_{i j} & =25 d_{i j} \\
\Delta t_{i j} & =25
\end{aligned}
$$

\section{Step 5}

Calculation of the price of ant footprint intensity between cities for the next cycle. The ant footprint intensity between cities on all trajectories between cities is likely to change due to evaporation and differences in the number of ants that pass through. For the next cycle, the ants that will pass through the path the price of the intensity has changed. The price of ant footprint intensity between cities for the next cycle is calculated by the equation:

$$
\begin{aligned}
& t_{i j}=\rho \cdot t_{i j}+\Delta t_{i j} \\
& t_{i j}=\rho \cdot t_{i j}+\Delta t_{i j} \\
& t_{i j}=0,5 \cdot 0,1+t_{i j} \\
& \Delta t_{i j}=0,05 \\
& \Delta t_{i j}=\frac{0,05}{0,1} \\
& \Delta t_{i j}=0,5
\end{aligned}
$$

\section{Conclusions}

In accordance with the discussion and trial results of the program carried out it can be concluded the antnet algorithm can be used to find the shortest route from a connectionless network. In the process of finding the shortest route on a connectionless network by knowing the nodes left by the ant. Antnet algorithm is very good in finding the shortest route on the network IP address.

\section{References}

[1] S. S. Dhillon and P. Van Mieghem, Performance analysis of the AntNet algorithm, Comput. Networks, vol. 51, no. 8, pp. 2104-2125, (2007).

[2] M. Chandana and S. Thakur, "Ant-Net: An adaptive routing algorithm," 1st IEEE Int. Conf. Power Electron. Intell. Control Energy Syst. ICPEICES 2016, (2017).

[3] B. Baran and R. Sosa, "AntNet: Routing Algorithm for Data Networks based on Mobile Agents," Intel. Artif., vol. 5, no. 12, pp. 75-84, (2001).

[4] W. Wang, F. Guo, F. Zheng, W. Tang, and J. Wang, "Research on routing protocols and simulation analysis for opportunistic networks," Int. J. Multimed. Ubiquitous Eng., vol. 10, no. 3, pp. 181-202, (2015). 
[5] S. Das, S. Subedi, N. Shekar, and V. Shet, "Network Performance Analysis of Dynamic Routing Protocols for Real Time Application," Www.Ijmer.Com |, vol. 4, pp. 49-57, (2014).

[6] S. Alamri, "An efficient shortest path routing algorithm for directed indoor environments," ISPRS Int. J. Geo-Information, vol. 7, no. 4, (2018).

[7] A. Fitro, O. Saeful Bachri, A. Ilham, S. Purnomo, and I. Frendianata, "Article ID: IJMET_09_02_078 Cite this Article: Achmad Fitro, Otong Saeful Bachri, Arif Ilham Sulistio Purnomo and Indra Frendianata, Shortest Path Finding in Geographical Information Systems using Node Combination and Dijkstra Algorithm," Int. J. Mech. Eng. Technol. (IJMET, vol. 9, no. 2, pp. 755-760, (2018).

[8] E. E. Ogheneovo and E. Seetam, "Open Access A Heuristic Graph-Based Shortest Path Algorithm for Optimizing Routing Problems American Journal of Engineering Research ( AJER )," no. 11, pp. 239-246, (2016).

[9] Sriadhi, et al. K-means method with linear search algorithm to reduce Means Square Error (MSE) within data clustering, Materials Science and Engineering 434 (2018) 012032. 